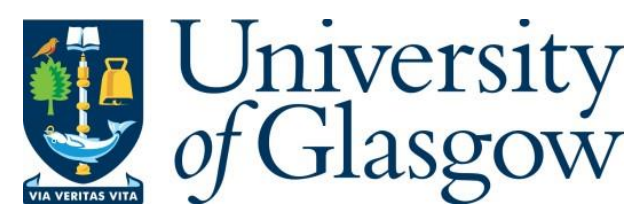

Coll, F. et al. (2018) Genome-wide analysis of multi- and extensively drug-resistant Mycobacterium tuberculosis. Nature Genetics, 50(2), pp. 307-316.

There may be differences between this version and the published version. You are advised to consult the publisher's version if you wish to cite from it.

http://eprints.gla.ac.uk/210147/

Deposited on: 14 February 2020

Enlighten - Research publications by members of the University of Glasgow http://eprints.gla.ac.uk 


\section{Genome-wide analysis of multi- and extensively drug-resistant Mycobacterium tuberculosis}

Francesc Coll ${ }^{1,}{ }^{*}$, Jody Phelan ${ }^{1 *}$, Grant A. Hill-Cawthorne ${ }^{2,3^{* *}}$, Mridul B Nair ${ }^{2, * *}$, Kim Mallard $^{1}$, Shahjahan Ali ${ }^{2}$, Abdallah M Abdallah², Saad Alghamdi ${ }^{4}$, Mona Alsomali², Abdallah O Ahmed ${ }^{5}$, Stephanie Portelli ${ }^{1}$, Yaa Oppong ${ }^{1}$, Adriana Alves ${ }^{6}$, Theolis Barbosa Bessa ${ }^{7}$, Susana Campino ${ }^{1}$, Maxine Caws ${ }^{8,9}$, Anirvan Chatterjee ${ }^{10}$, Amelia C Crampin ${ }^{11,12}$, Keertan Dheda ${ }^{13}$, Nicholas Furnham ${ }^{1}$, Judith R Glynn ${ }^{11,12}$, Louis Grandjean ${ }^{14}$, Dang Thi Minh Ha ${ }^{9}$, Rumina Hasan ${ }^{15}$, Zahra Hasan ${ }^{15}$, Martin L Hibberd ${ }^{1}$, Moses Joloba ${ }^{16}$, Edward C. Jones-López ${ }^{17}$, Tomoshige Matsumoto $^{18}$, Anabela Miranda ${ }^{6}$, David J Moore ${ }^{1,14}$, Nora Mocillo ${ }^{19}$, Stefan Panaiotov ${ }^{20}$, Julian Parkhill ${ }^{21}$, Carlos Penha22, João Perdigão ${ }^{23}$, Isabel Portugal ${ }^{23}$, Zineb Rchiad ${ }^{4}$, Jaime Robledo $^{24}$, Patricia Sheen ${ }^{13}$, Nashwa Talaat Shesha ${ }^{25}$, Frik A Sirgel ${ }^{26}$, Christophe Sola ${ }^{27}$, Erivelton de Oliveira Sousa ${ }^{28}$, Elizabeth M Streicher ${ }^{26}$, Paul Van Helden ${ }^{26}$, Miguel Viveiros ${ }^{29}$, Robert M Warren ${ }^{26}$, Ruth McNerney ${ }^{1,13,{ }^{* * *}, \text { Arnab Pain }}{ }^{2,30, * *}$, Taane G Clark ${ }^{1,11, * * *}$

1 Faculty of Infectious and Tropical Diseases, London School of Hygiene \& Tropical Medicine, Keppel Street, London, WC1E 7HT, United Kingdom

2 Pathogen Genomics Laboratory, BESE Division, King Abdullah University of Science and Technology (KAUST), Thuwal, Kingdom of Saudi Arabia

3 Sydney Emerging Infections and Biosecurity Institute and School of Public Health, Sydney Medical School, University of Sydney, NSW 2006, Australia

4 Laboratory Medicine Department, Faculty of Applied Medical Sciences, Umm Al-Qura University, Kingdom of Saudi Arabia

5 Department of Microbiology, Faculty of Medicine, Umm Al-Qura University, Makkah, Saudi Arabia 6 National Mycobacterium Reference Laboratory, Porto, Portugal

7 Centro de Pesquisas Goncalo Moniz, Fundacao Oswaldo Cruz Bahia R. Waldemar Falcao 121 Candeal 40296-710 Salvador Bahia Brazil

8 Liverpool School of Tropical Medicine, Pembroke Place, Liverpool, L3 5QA, United Kingdom 9 Pham Ngoc Thach Hospital for TB and Lung Diseases, Hung Vuong, Ho Chi Minh City, Vietnam 10 The Foundation for Medical Research, 84-A, R. G. Thadani Marg, Worli, Mumbai 400018, India 11 Faculty of Epidemiology and Population Health, London School of Hygiene \& Tropical Medicine, Keppel Street, London, WC1E 7HT, United Kingdom

12 Karonga Prevention Study, Malawi

13 Lung Infection and Immunity Unit, UCT Lung Institute, University of Cape Town, Groote Schuur Hospital, Observatory, 7925, Cape Town, South Africa. 
14 Laboratorio de Enfermedades Infecciosas, Laboratorios de Investigación y Desarrollo, Facultad de Ciencias y Filosofía, Universidad Peruana Cayetano Heredia, Lima, Peru

15 Department of Pathology and Laboratory Medicine, The Aga Khan University, Stadium Road, P.O. Box 3500, Karachi 74800, Pakistan

16 Department of Medical Microbiology, Makerere University College of Health Sciences, Kampala, Uganda

17 Section of Infectious Diseases, Department of Medicine, Boston Medical Center and Boston University School of Medicine, Boston, Massachusetts, USA

18 Osaka Anti-Tuberculosis Association Osaka Hospital, Osaka, Japan

19 Reference Laboratory of Tuberculosis Control, Buenos Aires, Argentina

20 National Center of Infectious and Parasitic Diseases, 1504 Sofia, Bulgaria

21 Wellcome Trust Sanger Institute, Hinxton, United Kingdom

22 Instituto Gulbenkian de Ciência, Lisbon, Portugal

23 iMed.ULisboa - Research Institute for Medicines, Faculdade de Farmácia, Universidade de Lisboa, Portugal

24 Corporación para Investigaciones Biológicas, Universidad Pontificia Bolivariana, Medellín,

Colombia

25 Regional Laboratory Directorate of Health Affairs, Makkah, Kingdom of Saudi Arabia.

26 Division of Molecular Biology and Human Genetics, SAMRC Centre for Tuberculosis Research,

DST/NRF Centre of Excellence for Biomedical Tuberculosis Research, Faculty of Medicine and Health

Sciences, Stellenbosch University, Tygerberg, South Africa

27 Institute for Integrative Cell Biology, CEA-CNRS-Université Paris-Saclay, Orsay, France

28 Centro de Pesquisas Goncalo Moniz, Fundacao Oswaldo Cruz Bahia R. Waldemar Falcao 121

Candeal 40296-710 Salvador Bahia Brazil and Laboratorio Central de Saude Publica Prof. Goncalo

Moniz Rua Waldemar Falcao, 123 Horto Florestal 40295-010 Salvador Bahia Brazil

29 Unidade de Microbiologia Médica, Global Health and Tropical Medicine, Instituto de Higiene e Medicina Tropical, Universidade Nova de Lisboa, UNL, Lisboa, Portugal

30 Global Station for Zoonosis Control, Global Institution for Collaborative Research and Education (GI-CoRE), Hokkaido University, N20 W10 Kita-ku, Sapporo, 001-0020 Japan

*Joint first authors, contributed equally.

${ }^{* *}$ Contributed equally.

*** Corresponding authors: Taane Clark (e-mail: taane.clark@lshtm.ac.uk) or Arnab Pain (email: arnab.pain@kaust.edu.sa), or Ruth McNerney (ruth.mcnerney@uct.ac.za) 
To further characterize the genetic determinants of resistance to anti-tuberculosis drugs we performed a genome-wide association study (GWAS) of 6,465 Mycobacterium tuberculosis clinical isolates from more than 30 countries. A GWAS approach within a mixed regression framework was followed by a phylogenetic-based test for independent mutations. In addition to established and recently described resistance genes, novel mutations were discovered for cycloserine, ethionamide and p-aminosalicylic acid. Capacity to detect resistance to ethionamide, pyrazinamide, capreomycin, cycloserine and para-aminosalicylic acid was enhanced by inclusion of insertions and deletions. Odds ratios for mutations within candidate genes were found to reflect levels of resistance. Novel epistatic relationships between candidate drug resistance genes were identified. Findings also suggest the involvement of efflux pumps ( $d r r A, R v 2688 c$ ) in the emergence of resistance. Findings from this study will inform the design of new diagnostic tests and expedite the investigation of resistance and compensatory epistatic mechanisms.

KEY WORDS: Mycobacterium tuberculosis, tuberculosis, GWAS, drug resistance, MDR-TB, XDR-TB

Word count: $\mathbf{4 , 2 0 0}$ 


\section{Introduction}

The emergence and spread of Mycobacterium tuberculosis (Mtb) resistant to multiple antituberculous drugs is of global concern. Programmatically incurable tuberculosis (TB), where effective treatment regimens cannot be provided due to resistance to the available drugs is a growing problem ${ }^{1}$. Resistance to rifampicin and isoniazid is classed as multidrug-resistant tuberculosis (MDR-TB), further resistance to the fluoroquinolones and any of the injectable drugs (amikacin, kanamycin or capreomycin) used to treat MDR-TB is termed extensively drug-resistant (XDR-TB). Treatment for patients with drug resistant tuberculosis is prolonged, expensive and outcomes are poor ${ }^{2}$. The drugs used are toxic and poorly tolerated, adverse events are common and may be severe and irreversible ${ }^{3}$. Inadequate treatment also risks amplification of resistance to further drugs and may prolong opportunities for transmission ${ }^{4}$.

Mtb has a clonal genome (size $4.4 \mathrm{Mb}$ ) with a low mutation rate and no evidence of betweenstrain recombination or horizontal gene transfer ${ }^{5}$. The Mtb complex comprises seven lineages, of which four are predominant in humans: Lineage 1, Indo-Oceanic (e.g. EastAfrican-Indian (EAI) spoligotype families); Lineage 2, East-Asian (e.g. W/Beijing spoligotype families); Lineage 3, East-African-Indian (e.g. Central-Asian-Strain (e.g. CAS-DELHI) spoligotype families) and Lineage 4, Euro-American (e.g. Latin American-Mediterranean (LAM), Haarlem and the "ill-defined" T spoligotype families) 5 .

Resistance in Mtb is mainly conferred by nucleotide variations (single nucleotide polymorphisms, insertions and deletions (indels)) in genes coding for drug-targets or converting enzymes. Changes in efflux pump regulation may have an impact on the emergence of resistance ${ }^{6}$ and putative compensatory mechanisms to overcome fitness 
impairment coincidental with the acquisition of resistance have been described for some drugs $^{7}$. Detection of resistance conferring mutations offers a means of rapidly identifying resistance to anti-tuberculosis drugs $^{8}$ but, with the exception of rifampicin, current molecular tests for resistance lack high levels of sensitivity ${ }^{8}$. To improve knowledge of genetic determinants of drug resistance we undertook whole genome analysis of a large collection $(n=6,465)$ of clinical isolates from more than 30 geographic locations, representing the four major Mtb lineages (Figure 1, Supplementary table 1). We adopted a GWAS approach to identify nucleotide variation and loci underlying drug resistance as successfully applied in Mtb $^{9-11}$ and other bacteria ${ }^{12,13}$. A total of 14 drugs with available phenotypic data on drug susceptibility testing were investigated (Supplementary table 2). Phenotypic drug susceptibility data was not available for each of the 14 drugs for every isolate and sample sizes ranged from over 6,000 for the most commonly tested first line drugs (isoniazid and rifampicin) to 255 and 248 for p-aminosalicylic acid and cycloserine, respectively, which are used to treat patients with XDR-TB. Here, we present findings from the most comprehensive study yet undertaken of the genetic determinants of resistance to anti-tuberculosis drugs or the Mtb resistome.

\section{RESULTS}

\section{Genetic diversity and drug resistance}

High quality genome-wide SNPs $(102,160)$, indels $(11,122)$, and large deletions $(284)$ were identified across all samples $(n=6,465)$. Most SNPs $(93.1 \%)$ had rare minor alleles (allele frequency <1\%) (Supplementary Figure 1). Similarly, small indels were rare $(96.6 \%$ had frequency $<1 \%$ ), and ranged in size from 1 to $45 \mathrm{bp}$. A phylogenetic tree and principal 
component analysis constructed using all genome-wide SNPs revealed the expected clustering by lineage (Figure 2, Supplementary Figure 2 ).

Phenotypic analysis of susceptibility to anti-tuberculosis drugs found $31.2 \%$ of isolates were resistant to at least one drug, $15.1 \%$ were categorized as MDR-TB and $4.3 \%$ as XDR-TB (Supplementary table 2, Figure 2). Fourteen drugs were included in the genome-wide analysis: isoniazid (INH), rifampicin (RIF), ethionamide (ETH), pyrazinamide (PZA), ethambutol (EMB), streptomycin (STM), amikacin (AMK), capreomycin (CAP), kanamycin (KAN), ciprofloxacin (CIP), ofloxacin (OFL), moxifloxacin (MOX), cycloserine (CYS) and paraaminosalicylic acid (PAS). Drug family groups including the second-line injectable drugs (SLID: AMK, KAN, CAP) and fluoroquinolones (FLQ: CIP, OFL, MOX) were also analysed. Insufficient phenotypic data was available for the inclusion of the new and repurposed drugs, bedaquiline, delamanid and linezolid. To reveal loci associated with drug resistance complementary methods were applied to mutations and aggregated non-synonymous mutations: a tree-based "PhyC" test for convergent evolution to detect homoplastic variants9 and a GWAS approach within a mixed regression framework (See Online methods). Unless stated otherwise, all analysis used the complete dataset. First, we consider MDR-TB and XDRTB phenotypes (Table 1) and then individual drug GWAS and evolutionary results (Table 2).

\section{GWAS and phyC tests for MDR-TB and XDR-TB}

The gene-based GWAS of MDR-TB versus susceptible identified $r p o B$ (RIF), Rv1482c-fabG1 operon (INH, ETH), inhA (INH, ETH), katG (INH), and oxyR'-ahpC (compensatory mechanism for INH). The katG mutations at codon 315 (S315T, S315N, S315R) were all statistically significant, and collectively were the most frequent mutations (75.2\%) across all resistance 
loci identified, consistent with a recent study ${ }^{14}$ and highlighting their pivotal role in the emergence of INH resistance and MDR-TB. The katG S315T mutation is thought to emerge before RIF resistance associated mutations and therefore, from an evolutionary standpoint, preclude the emergence of MDR-TB ${ }^{14,15}$. However, our analysis highlighted that Rv1482cfabG1 and inhA mutations, in the absence of katG S315T, can emerge prior to MDR-TB, as previously shown in two phylogenetically-independent clades in Lisbon ${ }^{16,17}$. The other frequent MDR-TB mutations in our study included rpoB-S450L (RIF, 64.2\%), embB-M306L/V/I (EMB, 49.1\%), and rpsL-K43R (STM, 42.2\%) (Supplementary table 3), and the magnitude correlates with historical treatment practice and emergence of resistance. There are corresponding signals of INH/RIF co-resistance with other first-line drugs, with the detection of gene-based association signals for gid (STM) and rpsL (STM), and a SNP-based association signal for the embC-embA intergenic region (EMB). SNP-based PhyC analysis detected the above loci, but in addition folC (PAS), pncA-Rv2044c intergenic region (PZA), and whiB6Rv3863 intergenic (putative STM or ETH) regions.

The gene-based GWAS of XDR-TB versus MDR-TB identified mutations in gyrA (FLQ), rrs (aminoglycosides), the embC-embA intergenic region and ubiA (EMB). The PhyC test additionally revealed eis- $R v 2417 c$ (KAN), gyrB (FLQ), rrs (aminoglycosides), folC (PAS), alr (CYS), gid (STM) SNPs, and a novel mutation in the thyX-hsdS.1 intergenic region (A-9T, PAS) ${ }^{18,19}$. In addition to loci identified above, the gene-based GWAS comparing XDR-TB to susceptible groups identified rpoC (a compensatory mechanism for RIF resistance), ethA (ETH), eis-Rv2417c (KAN) and PPE52-nuoA (novel intergenic region, G-314T). The PhyC test additionally detected SNPs in gyrB (FLQ, D461N, D641H, T500N, T500I and A504V), supported 
the thyX-hsdS.1 intergenic region SNP finding (PAS, A-9T), as well as identified a previously unreported ubiA SNP association (EMB, M180V).

The drrA Arg262Gly mutation was significantly associated with XDR-TB compared to susceptible (mutation frequency $18 \%$ vs. $0 \%$, respectively, $\mathrm{P}=1.5 \times 10^{-8}$ ). We hypothesize that drrA may be involved in export of drugs across the membrane based on its strong association with XDR-TB in our study and its functional annotation as a probable transporter of antibiotics across the membrane (TubercuList, see URLs). This hypothesis is in accordance with the findings that $r p o B$ mutations in Mtb may trigger compensatory transcriptional changes in secondary metabolism genes, in particular, in the biosynthesis and export of phthiocerol dimycocerosate (PDIM), increasing its expression and activity. As a consequence these strains became more virulent and multidrug resistant, increasing their fitness by increased efflux activity and lipid metabolism ${ }^{20,21}$. Similarly, a mutation in the Rv1144-mmpL13a intergenic region (C-102A) was highly associated with XDR-TB versus susceptible (mutation frequency $17 \%$ vs. $0 \%$, respectively, $\left.\mathrm{P}=1.5 \times 10^{-7}\right)$. This mutation sits in the promoter to the operon containing $m m p L 13 a$ and $m m p L 13 b$, which code for transmembrane transport proteins and could influence expression of these proteins ${ }^{6}$.

\section{Lineage-specific and compensatory mechanisms}

We conducted a stratified GWAS per lineage to identify lineage-specific loci associated with drug resistance. Most associations were present in more than one lineage. The largest number of lineage-specific drug resistance mutations were found in lineage 4 , which was the largest collection investigated and contained more genetically diverse clones ${ }^{5}$, implying that geographically restricted mutations are being captured (Supplementary table 4). A previously 
unreported putative compensatory locus was identified for pyrazinamide (pncB1) through analysis of lineage 1 which reached borderline significance for lineage 3.

We applied a systematic approach to reveal epistatic interactions between GWAS loci (from Table 2) or explore known compensatory effects using a test of non-random association to detect the frequent co-occurrence of mutations in pairs of loci (Fisher exact test, P-value cutoff $<1 \times 10^{-8}$ ) (Supplementary table 5 ). Deep phylogenetic mutations were removed to increase robustness. This approach proved to be successful at identifying well-known compensatory relationships between $r p o B$ and $r p o C$ loci (RIF) ${ }^{7}, r p o B$ and $r p o A(\mathrm{RIF})^{22}$ and $k a t G$ and $\operatorname{xxy} R^{\prime}-a h p C(\mathrm{INH})^{23}$. We captured the frequent co-occurrence of embB and ubiA mutations which together are known to lead to high levels of EMB resistance ${ }^{24}$, and they are therefore unlikely to represent a compensatory mechanism. Novel epistatic relationships included pncA with pncB2 (PZA) and thyA with thyX-hsdS.1 (PAS). The pncB2 effect appears to be specific to lineage 4 (Supplementary table 6). The other nicotinamide co-factor, pncB1, had weaker evidence of an epistatic relationship with pncA in lineage $1(P=0.0016)$ (Supplementary table 6). Similarly, there was marginal evidence for pyrG (lineage $4, P=0.00016)^{25}$ and $R v 0565 c$ (lineage 2, $\mathrm{P}=0.00027$ ) with ethA $(\mathrm{ETH})^{26}$ (Supplementary table 6). Follow-up investigations will need to determine whether mutations in these loci have an impact on the minimal inhibitory concentration (MIC) values or function as compensatory mechanisms.

Overall, the GWAS approach was effective at detecting known drug resistance determinants and epistatic (gene-gene) relationships and identified novel ones that warrant functional validation in future studies. As resistance loci for individual drugs, especially second-line treatments, may be masked by an analysis of the composite MDR-TB and XDR-TB outcomes, 
we repeated the GWAS, PhyC test and epistatic analysis for the 14 individual drugs considered.

\section{GWAS and phyC tests for individual drugs}

Rifampicin, isoniazid and ethionamide

The $r p o B$ locus showed the strongest association with RIF resistance, but the compensatory effects of $r p o C$ and $r p o A$ were also evident through homoplasy SNP analysis. As previously reported non-synonymous SNPs in rpoC (272 identified) were spread across the whole gene ${ }^{27}$. Altered or diminished activity of the catalase-peroxidase enzyme KatG is the most frequent mechanism of isoniazid resistance ${ }^{28}$, and as expected, the katG gene ranked first in the GWAS for this drug. Mutations in proposed INH drug targets, kas $A$ and kasB previously included in some drug resistance databases, did not reach statistical significance in our study ${ }^{29}$, suggesting an odds ratio below our detection level of 1.4 (with 99\% confidence of detection, $90 \%$ statistical power). Both inhA, encoding the molecular target of isoniazid ${ }^{30}$ and the $R v 1482 c-f a b G 1$ intergenic region harbouring its promoter, showed strong associations with INH and ETH, with greater effects in the former. In addition, oxy $R^{\prime}-a h p C$ intergenic associated mutations (20 detected) were found in the presence of katG polymorphisms (28), supporting its role as a compensatory mechanism. For ethionamide, the ethA locus, encoding the drugmetabolising enzyme was found to be associated with resistance as described previously ${ }^{31}$. A total of 153 non-synonymous mutations were identified in ethA, scattered throughout the gene and mostly affecting codons different from those already described ${ }^{8}$.

\section{Ethambutol}

Mutations in the embCAB operon, which encodes for enzymes involved in the biosynthesis of arabinan components of the mycobacterial cell wall, are mostly responsible for EMB 
resistance but are not fully penetrant for resistance ${ }^{32}$. The embB and the embC-embA intergenic region had the strongest associations. $R v 3806 c$ (ubiA), described to contribute to high levels of $E M B$ resistance in vitro ${ }^{17}$ was also significantly associated in our analysis demonstrating a role in clinical samples too across all four lineages. Two novel loci were identified: Rv2820c thought to enhance mycobacterial virulence ex vivo and in vivo, and Rv3300c a conserved protein with unknown function (TubercuList, see URLs).

\section{Pyrazinamide}

The pncA locus was the highest ranked association with PZA resistance in the GWAS and was a target of independent mutation, consistent with its established role ${ }^{33}$. Additionally, many low frequency SNPs were reported which were not used in the association analysis and could potentially confer resistance (Supplementary data 1). Other proposed PZA targets, namely $r p s A^{34}$ and panD $D^{35}$, did not reach statistical significance in the GWAS and were not targets of independent mutation among PZA resistant strains in our collection.

\section{Streptomycin}

The rpsL, rrs and gid loci, all known to be involved in STM resistance ${ }^{18}$ were identified by GWAS. Mutations in rpsL are known to lead to intermediate to high levels of STM resistance ${ }^{36}$, and accordingly we observed high odds ratios indicative of high penetrance in association signals in this locus (Figure $\mathbf{3 A}$ ). In contrast, candidate rrs and gid gene polymorphisms showed weaker overall signals (lower odds ratio) in the GWAS, which concurs with existing evidence that gid and rrs mutations confer lower levels of resistance ${ }^{36}$ (differences in odds ratios: $r p s /$ vs. $r r s /$ gid Wilcoxon $\mathrm{P}=0.03$; rps/ vs. gid Wilcoxon $\mathrm{P}=0.04)$.

\section{Fluoroquinolones and Second-line injectables}

The gene- and SNP-based GWAS analysis revealed the gyrA locus, which encodes for the molecular target of $\mathrm{FLQ}^{37}$, as the strongest association signal. In addition to homoplastic 
mutations in $g y r A$, evidence of independent mutation was detected in $g y r B^{38}$. The $R v 2688 \mathrm{c}$ C213R mutation was associated with MOX and FLQ resistance but did not reach statistical significance in OFL. The antibiotic transport ATP-binding protein encoded by $R v 2688 \mathrm{c}$ is a known FLQ efflux gene ${ }^{39}$. As expected the strongest resistance gene and SNP-based association signals across AMK, KAN, and CAP was with the aminoglycoside (SLID) target gene $r r s^{18}$. Association was observed with mutations in the eis promoter known to result in low levels of KAN resistance but not in co-resistance with other aminoglycosides ${ }^{40}$. Although the eis promoter mutations had a lower median odds ratio than that of rrs mutations, potentially supporting evidence that $r$ rs mutations confer higher levels of KAN resistance ${ }^{40}$, this was not statistically significant due to small sample size (differences in odds ratios Wilcoxon $P=0.24$ ) (see Figure 3A).

\section{D-Cycloserine}

CYS inhibits the Alr enzyme, responsible for the conversion of L-Alanine into D-Alanine, by competing with L-Alanine for the active site. Resistance to CYS results from mutations in the alr coding region ${ }^{41}$. In our study alr was significantly associated with CYS resistance (Table 2) in line with recent evidence showing that clinical strains with alr mutations exhibit increased resistance to $\mathrm{CYS}^{11}$ and harboured multiple homoplastic mutations including Phe4Leu, Lys113Arg and Met343Thr. In a previous study, the Met343Thr mutation was detected in an XDR-TB strain that had been exposed to CYS treatment, predicted to alter the protein structure of Alr, and therefore it was hypothesised to be involved in CYS resistance ${ }^{42}$. To further understand the functional impact of the mutations found in alr we modelled the effect of these variants using the available crystal protein structure (PDB 1XFC, Supplementary figure 3). Mutations in alr were found to differ in their proximity to the CYS binding site and their effect on protein stability and ligand binding (Supplementary table 7). The Met343Thr 
mutation (found in 12 susceptible and 2 resistant isolates) was predicted to have more drastic effect on protein structure compared to Lys113Arg, the most frequent mutation among CYS resistant isolates (in 7 susceptible and 23 resistant isolates). There appears to be a balance between the fitness cost associated with mutations and their frequency (Supplementary table 7). The Met343Thr mutation appears independently throughout the phylogenetic tree, but did not reach statistical significance for association to drug resistance (XDR-TB or CYS), implying that selection may be acting on this mutation but drug resistance may not be the driving factor.

\section{Para-aminosalicylic acid}

PAS is a pro-drug that is converted into its active form by thy $A$ - a thymidylate synthase, which is an essential gene for Mtb survival. The candidate drug resistance loci are those involved in folate metabolism and biosynthesis of thymidine nucleotides (thy $A, d f r A$, folC, folP1, folP2 and thyX $X)^{19}$. Of these, thyA and thyX-hsdS.1 (directly upstream of thyX) and were found to be associated with PAS drug resistance in both gene- and SNP-based GWAS. Importantly, it has been shown that G-16A SNP found in our study increased thyX expression by 18 -fold relative to wild-type promoter although no link with PAS resistance was made ${ }^{18}$. Of 3 PAS resistance strains with the G-16A thyX promoter mutation, 2 also had a thyA mutation (P145L, H207R), further supporting that up-regulation of thyX is involved in resistance to PAS ${ }^{26}$, or has a compensatory role. The G-16A thyX is a homoplastic mutation, and therefore more likely to be compensatory.

Overall, the ( $\log )$ odds ratios for mutations with known levels of resistance based on MIC values followed an increasing trend from low to intermediate to high levels (Figure 3B; log odds ratios: linear regression trend $P=1.5 \times 10^{-9}$, high vs. intermediate $P=5.2 \times 10^{-5}$; 
intermediate vs. low $\left.\mathrm{P}=5.8 \times 10^{-10}\right)$. This analysis demonstrates a potential utility of using odds ratios and their statistical significance to indicate the impact of a mutation and its propensity to cause low, intermediate or high-level resistance. Further, the odds ratios for the novel findings were marginally lower than those for known ones (Wilcoxon test $P=8.3 \times 10^{-5}$ ), reflecting the ability of the GWAS to discover effect sizes of lower magnitude (Figure $\mathbf{3 C}$ ). A pathway analysis comparing MDR-TB/XDR-TB to susceptible strains revealed only one significant annotation cluster with 17.7-fold enrichment for antibiotic resistance and response to antibiotics $\left(P=1.6 \times 10^{-7}\right)$, further confirming the robustness of the GWAS approach.

\section{Association tests using small indels and large deletions}

An analysis of genome-wide small indels revealed associations in candidate resistance genes and operons (Supplementary table $\mathbf{8}$, Supplementary data $\mathbf{1}$ ). The candidate genes differed in their abundance of small indels, reflecting their essentiality for survival: drug targets had less density of indels whereas drug-metabolising enzymes had a greater density. For example, the pncA gene was the most polymorphic coding region (PZA, 44.72 indels $/ k b$ ) while the least polymorphic was $r p o B$ (RIF, 2.3 indels / kb). Although, most small indels (83\%) in the candidate regions were $1 \mathrm{bp}$ in length and caused frame-shifts, the indels in $r p o B$ inserted or deleted whole codons, i.e. they did not cause a shift in the codon reading frame. Indels in $r p o B, p n c A$ and the embAB promoter region were associated with MDR-TB, XDR-TB and their respective targets/activators. Indels in ethA were associated with ETH and XDR-TB resistance. Similarly, gid indels were associated with STM as expected. 
The analysis of CYS revealed indel associations with the ald gene, supporting recent reports that loss of function in ald confers resistance ${ }^{11}$. Thus resistance to CYS appears to be conferred by both SNPs in alr and indels in ald. Indels found in rrs were associated with KAN and CAP resistance, however they did not reach statistical significance for STM, which has a different drug binding site. CAP resistance was also found to be associated with three indels in tlyA, two of which are located at the $3^{\prime}$ end of the gene. In general, indels were distributed throughout the gene lengths however there was some evidence of areas of higher density such as the pncA region between codons 130 and 132 (close to the catalytic centre) and the rрoB 427-434 codon region.

The only large deletion association identified by GWAS was a region encompassing the thy $A$ and $d f r A$ genes and PAS resistance. Five samples across 4 countries contained large thyA- $d f r A$ deletions of varying length (Supplementary table 9, Supplementary figure 4). Associations in partial or whole gene deletions in $k a t G$, ethA and $p n c A$, were close to statistical significance $(P<0.05)$. These genes activate pro-drugs, and none are considered to be essential to Mtb survival. The large deletions detected occur independently in different branches of the phylogenetic tree and are likely to offer an alternative route to resistance compared to small genomic variants, across lineages and populations.

\section{Effects on resistance prediction using GWAS variants}

We sought to establish if any of the mutations found in association and homoplastic analysis increased the predictability of individual drug resistance phenotypes (Table 3). We used the reported phenotypic drug susceptibility test result as the reference standard to calculate the sensitivity and specificity for mutation-resistance predictions. Using a previously established 
library of mutations ${ }^{8,17}$ (TBDR library), we found that although the sensitivity was greater than $80 \%$ in $8 / 14$ drugs, a substantial proportion of resistance phenotypes were not explained by known mutations, particularly in second-line drugs. Using the novel SNPs identified in this study we gained sensitivity for PAS (+10\%), ETH $(+14 \%)$ and CYS $(+50 \%$, not included in the TBDR library) (Table 3). The additional inclusion of small indels and large deletions further improved the predictive ability for 9 drugs while maintaining specificities of at least $90 \%$, except for ETH which is $72 \%$ (Table 3 ).

\section{DISCUSSION}

To provide genomic insights into Mtb drug resistance we have combined the power of whole genome sequencing with a genome-wide association analytical approach in the largest and most geographically widespread study to date, encompassing a total of 6,465 clinical isolates of Mtb from more than 30 countries. Large sample sizes are required to identify complex or infrequent genetic effects, but also to negate effects due to possible errors in phenotypic drug susceptibility testing and misclassification ${ }^{43}$. The lack of standardization of phenotypic testing methodologies for Mtb is also a potential source of bias which was reduced by the inclusion of samples from different countries and laboratories using a variety of quality assured testing methodologies. Whilst resistant phenotypes may be imputed from established resistance causing mutations, inferring susceptibility to a drug cannot be assumed in the absence of corroborating evidence ${ }^{17}$. The completeness of our susceptibility test data meant that both GWAS and homoplasy-based methods could be applied across 14 drugs.

The GWAS identified well-established resistance loci and compensatory relationships, thereby confirming the authenticity and robustness of the approach. It also revealed several 
recently discovered loci (folC, ubiA, thyX-hsdS.1, thyA, alr, ald, dfrA-thyA), new epistatic relationships ( $p n c A$ with pncB2, and thyA with thyX-hsdS.1) and efflux pumps represented by the $A B C$ transporters $d r r A$ and $R v 2688 c$ associated with drug resistance. The novel genetic markers associated with resistance identified in this GWAS included SNPs in the ethA and thyX promoters, small indels in pncA and ald, and large deletions in pro-drug activators such as ethA and katG. These loci warrant functional follow-up and characterization studies to fully elucidate their role in treatment failure. The associations identified may shed light on the molecular mechanisms underlying drug resistance and assist in the design of novel antibiotics.

In our study, sample sizes for second-line drugs were reduced compared to the first-line drugs. This was due to the lower prevalence of resistance to second-line drugs and the fact that isolates susceptible to first-line drugs are not routinely tested for second-line drugs. However, due to the large effect that causal mutations have on drug resistance phenotypes, although not ideal, relatively small samples of bacterial genomes can be sufficient to identify causal mutations ${ }^{43}$ as has been demonstrated in previous studies on $\mathrm{Mtb}{ }^{10-12}$. It should be noted that bedaquiline, delamanid and linezolid were excluded from our analysis due to the paucity of phenotypic susceptibility data.

The analysis also highlighted the importance of indels on drug resistance, particularly their high density in drug-metabolizing genes, in contrast to highly essential drug-target genes where their density was low. The inclusion of small indels and large deletions improved the predictability of resistance phenotypes. However, for drugs like CYS and PAS mechanisms of drug resistance remain unknown and larger numbers of resistant cases will be required to 
elucidate them. It is also possible that unknown mechanisms may be explained by the role of epigenetics and gene expression ${ }^{44}$.

Mtb strains are usually classified as drug resistant or susceptible based on their capacity to grow in vitro when exposed to a critical concentration of the drug. Phenotypic testing methods have a degree of uncertainty, especially close to the threshold ${ }^{43}$. Testing against a range of drug concentrations to establish the minimum inhibitory concentration (MIC) is a preferred approach to determine the level of resistance but is not routinely undertaken ${ }^{40}$. MIC values were not available for every isolate presented here, but despite this limitation, loci known to be involved in low-levels of resistance (Table 3), were identified by our analysis. Indeed, our analysis revealed a relationship between known levels of resistance and the odds ratios from the GWAS, which could aid the clinical interpretation of molecular diagnostic data including measuring the sensitivity and specificity of individual mutations when diagnosing drug resistance.

Emergence of resistance is driven by drug exposure and local TB treatment practices are a major influence on the prevalence and pattern of resistance. A limitation of this study was the sampling methodology since collection of the isolates was not controlled or systematic and resistant isolates were not evenly distributed across collection sites. However, within our study population we covered the four major Mtb lineages across 5 continents and sampled multiple geographical regions, allowing us to observe differences in the prevalence of drug resistance mutations and mechanisms. Some of drug resistance and compensatory/epistatic relationships were found to vary across geographical populations and bacterial lineage, implying that regional variation should be considered to fully characterise genotype- 
phenotype relationships. The differential lineage effects could impact on relative virulence between strain-types. Enhanced understanding of the genetic basis of anti-tuberculous phenotypic drug resistance will also aid in the development of more accurate molecular diagnostics for drug-resistant TB. An important finding of this study is the significance of genomic variation other than SNPs which has implications for the design of molecular tests for resistance. Improved tools are needed to guide treatment of patients with multidrugresistant disease where personalized treatment offers improved rates of cure ${ }^{45}$. Next generation sequencing offers a comprehensive assessment and may be used to guide treatment ${ }^{45}$. Although such technology is currently being implemented in some low burden countries such as the United Kingdom, it remains to be trialled in resource-poor settings that are representative of most TB patients worldwide.

\section{ACKNOWLEDGMENTS}

The project was supported by the KAUST faculty baseline research fund (KAUST-BRFBAS/1/1020-01-01) to AP. The authors wish to thank members of KAUST Bioscience Core laboratory who sequenced samples. We thank the Wellcome Trust Sanger Institute core and pathogen sequencing and informatics teams who were involved in the Malawi and Uganda studies. The work was funded in part by the Wellcome Trust (Grant numbers WT096249/Z/11/B, WT088559MA, WT081814/Z/06/Z, and WT098051), and the Wellcome Trust-Burroughs Wellcome Fund Infectious Diseases Initiative grant (number 063410/ABC/00/Z). FC was the recipient of a Bloomsbury College PhD Studentship and was supported by the Wellcome Trust (201344/Z/16/Z); JPe received a Fundação para a Ciência e

a Tecnologia (Portugal) Post-doctoral fellowship fund (SFRH/BPD/95406/2013). The Calouste Gulbenkian Foundation, the Institute Gulbenkian in Lisbon and European Society of Clinical 
Microbiology and Infectious Diseases supported the research of CP, JPe, IP and MV. JPh is funded by a BBSRC PhD studentship. TC is funded by the Medical Research Council UK (Grant no. MR/K000551/1 and MR/M01360X/1, MR/N010469/1, MC_PC_15103). TM is supported by the Ministry of Health, Labor and Welfare of Japan (H21- Shinkou-Ippan-008 and H24Shinkou-Ippan-010). We thank Nerges Mistry (Foundation for Medical Research, Mumbai) for contributing M. tuberculosis archived strains and drug-sensitivity testing data. We wish to thank Prof. Goncalo Moniz at the Laboratorio Central de Saude Publica for supporting the collection of samples in Brazil, and the South African National Health Laboratory Service for their contribution providing access clinical Mtb isolates. The MRC eMedLab computing resource was used for bioinformatics and statistical analysis. The authors declare no conflicts of interest. The work has been performed as part of the TB Global Drug Resistance Collaboration (see URLs).

\section{AUTHOR CONTRIBUTIONS}

$\mathrm{RM}, \mathrm{AP}$ and TC conceived and directed the project. GH, KM and RM coordinated sample collection and undertook DNA extraction. SAI, AOA, AA, TB, MC, AC, ACC, KD, LG, JG, DH, RH, $Z H, P H, M J, E J, T M, A M, N M, D M, S P, I P, C P, J P e, J R, P S, N S, F S, C S, E O S, E S, N P, M V$ and RW undertook sample collection, DNA extraction, genotyping and phenotypic drug resistance testing. GH, MBN, MAS, ZR and SA prepared libraries for Illumina sequencing. JPa led the generation of Malawian and Ugandan sequencing data. FC and JPh performed bioinformatic and statistical analyses under the supervision of TC. SP and YO performed additional confirmatory analysis under the supervision of $\mathrm{MH}, \mathrm{NF}$ and TC. FC, JPh, SP, SC, NF, MH, RM, AP, and TC interpreted results. FC, JPh, RM and TC wrote the first draft of the manuscript. All 
authors commented and edited on various versions of the draft manuscript. FC, JPh, RM, and

TC compiled the final manuscript. All authors approved the final manuscript.

\section{COMPETING FINANCIAL INTERESTS STATEMENT}

There are no conflicts of interest.

\section{REFERENCES}

1. Dheda, K. et al. Global control of tuberculosis: from extensively drug-resistant to untreatable tuberculosis. The Lancet Respiratory Medicine 2, 321-338 (2014).

2. Bastos, M. L. et al. Treatment Outcomes of Patients With Multidrug-Resistant and Extensively Drug-Resistant Tuberculosis According to Drug Susceptibility Testing to First- and Second-line Drugs: An Individual Patient Data Meta-analysis. Clinical Infectious Diseases 59, 1364-1374 (2014).

3. Shean, K. et al. Drug-Associated Adverse Events and Their Relationship with Outcomes in Patients Receiving Treatment for Extensively Drug-Resistant Tuberculosis in South Africa. PLoS ONE 8, e63057 (2013).

4. Clark, T. G. et al. Elucidating Emergence and Transmission of Multidrug-Resistant Tuberculosis in Treatment Experienced Patients by Whole Genome Sequencing. PLoS ONE 8, e83012 (2013).

5. Coll, F. et al. A robust SNP barcode for typing Mycobacterium tuberculosis complex strains. Nature Communications 5, 4812 (2014).

6. Black, P. a et al. Energy Metabolism and Drug Efflux in Mycobacterium tuberculosis. Antimicrobial Agents and Chemotherapy 58, 2491-2503 (2014).

7. de Vos, M. et al. Putative Compensatory Mutations in the rpoC Gene of RifampinResistant Mycobacterium tuberculosis Are Associated with Ongoing Transmission. Antimicrobial Agents and Chemotherapy 57, 827-832 (2013).

8. Coll, F. et al. Rapid determination of anti-tuberculosis drug resistance from wholegenome sequences. Genome Medicine 7, 51 (2015).

9. Farhat, M. R. et al. Genomic analysis identifies targets of convergent positive selection in drug-resistant Mycobacterium tuberculosis. Nature Genetics 45, 11831189 (2013).

10. Zhang, H. et al. Genome sequencing of 161 Mycobacterium tuberculosis isolates from China identifies genes and intergenic regions associated with drug resistance. Nature Genetics 45, 1255-1260 (2013).

11. Desjardins, C. A. et al. Genomic and functional analyses of Mycobacterium tuberculosis strains implicate ald in D-cycloserine resistance. Nature Genetics 48, 544-551 (2016).

12. Earle, S. G. et al. Identifying lineage effects when controlling for population structure improves power in bacterial association studies. Nature Microbiology 1, 16041 (2016). 
13. Chewapreecha, C. et al. Comprehensive identification of single nucleotide polymorphisms associated with beta-lactam resistance within pneumococcal mosaic genes. PLoS genetics 10, e1004547 (2014).

14. Manson, A. L. et al. Genomic analysis of globally diverse Mycobacterium tuberculosis strains provides insights into the emergence and spread of multidrug resistance. Nature Genetics 49, 395-402 (2017).

15. Cohen, K. a. et al. Evolution of Extensively Drug-Resistant Tuberculosis over Four Decades: Whole Genome Sequencing and Dating Analysis of Mycobacterium tuberculosis Isolates from KwaZulu-Natal. PLOS Medicine 12, e1001880 (2015).

16. Perdigão, J. et al. Unraveling Mycobacterium tuberculosis genomic diversity and evolution in Lisbon, Portugal, a highly drug resistant setting. BMC genomics 15, 991 (2014).

17. Phelan, J. et al. The variability and reproducibility of whole genome sequencing technology for detecting resistance to anti-tuberculous drugs. Genome Medicine 8, 132 (2016).

18. Meier, A., Sander, P., Schaper, K. J., Scholz, M. \& Böttger, E. C. Correlation of molecular resistance mechanisms and phenotypic resistance levels in streptomycinresistant Mycobacterium tuberculosis. Antimicrobial agents and chemotherapy 40, 2452-4 (1996).

19. Zhang, X. et al. Genetic Determinants Involved in $p$-Aminosalicylic Acid Resistance in Clinical Isolates from Tuberculosis Patients in Northern China from 2006 to 2012. Antimicrobial Agents and Chemotherapy 59, 1320-1324 (2015).

20. Bisson, G. P. et al. Upregulation of the phthiocerol dimycocerosate biosynthetic pathway by rifampin-resistant, rpoB mutant Mycobacterium tuberculosis. Journal of bacteriology 194, 6441-52 (2012).

21. Chatterjee, A., Saranath, D., Bhatter, P. \& Mistry, N. Global transcriptional profiling of longitudinal clinical isolates of Mycobacterium tuberculosis exhibiting rapid accumulation of drug resistance. PloS one 8, e54717 (2013).

22. Comas, l. et al. Whole-genome sequencing of rifampicin-resistant Mycobacterium tuberculosis strains identifies compensatory mutations in RNA polymerase genes. Nature Genetics 44, 106-110 (2011).

23. Sherman, D. R. et al. Compensatory ahpC Gene Expression in Isoniazid-Resistant Mycobacterium tuberculosis. Science 272, 1641-1643 (1996).

24. Safi, H. et al. Evolution of high-level ethambutol-resistant tuberculosis through interacting mutations in decaprenylphosphoryl- $\beta$-D-arabinose biosynthetic and utilization pathway genes. Nature genetics 45, 1190-1197 (2013).

25. Mori, G. et al. Thiophenecarboxamide Derivatives Activated by EthA Kill Mycobacterium tuberculosis by Inhibiting the CTP Synthetase PyrG. Chemistry \& Biology 22, 917-927 (2015).

26. Merker, M. et al. Whole genome sequencing reveals complex evolution patterns of multidrug-resistant Mycobacterium tuberculosis Beijing strains in patients. PloS one 8, e82551 (2013).

27. Casali, N. et al. Evolution and transmission of drug-resistant tuberculosis in a Russian population. Nature genetics 46, 279-86 (2014).

28. Zhang, Y., Heym, B., Allen, B., Young, D. \& Cole, S. The catalase-peroxidase gene and isoniazid resistance of Mycobacterium tuberculosis. Nature 358, 591-593 (1992).

29. Larsen, M. H. et al. Overexpression of inhA, but not kasA, confers resistance to 
isoniazid and ethionamide in Mycobacterium smegmatis, $M$. bovis BCG and $M$. tuberculosis. Molecular microbiology 46, 453-66 (2002).

30. Banerjee, A. et al. inhA, a gene encoding a target for isoniazid and ethionamide in Mycobacterium tuberculosis. Science 263, 227-230 (1994).

31. DeBarber, a E., Mdluli, K., Bosman, M., Bekker, L. G. \& Barry, C. E. Ethionamide activation and sensitivity in multidrug-resistant Mycobacterium tuberculosis. Proceedings of the National Academy of Sciences of the United States of America 97, 9677-82 (2000).

32. Telenti, A. et al. The emb operon, a gene cluster of Mycobacterium tuberculosis involved in resistance to ethambutol. Nature Medicine 3, 567-570 (1997).

33. Scorpio, A. \& Zhang, Y. Mutations in pncA, a gene encoding pyrazinamidase/nicotinamidase, cause resistance to the antituberculous drug pyrazinamide in tubercle bacillus. Nature Medicine 2, 662-667 (1996).

34. Shi, W. et al. Pyrazinamide Inhibits Trans-Translation in Mycobacterium tuberculosis. Science 333, 1630-1632 (2011).

35. Shi, W. et al. Aspartate decarboxylase (PanD) as a new target of pyrazinamide in Mycobacterium tuberculosis. Emerging Microbes \& Infections 3, e58 (2014).

36. Perdigão, J. et al. GidB mutation as a phylogenetic marker for Q1 cluster Mycobacterium tuberculosis isolates and intermediate-level streptomycin resistance determinant in Lisbon, Portugal. Clinical Microbiology and Infection 20, 0278-0284 (2014).

37. Takiff, H. E. et al. Cloning and nucleotide sequence of Mycobacterium tuberculosis gyrA and gyrB genes and detection of quinolone resistance mutations. Antimicrobial Agents and Chemotherapy 38, 773-80 (1994).

38. Kocagöz, T. et al. Gyrase mutations in laboratory-selected, fluoroquinolone-resistant mutants of Mycobacterium tuberculosis H37Ra. Antimicrobial Agents and Chemotherapy 40, 1768-74 (1996).

39. Pasca, M. R. et al. Rv2686c-Rv2687c-Rv2688c, an ABC Fluoroquinolone Efflux Pump in Mycobacterium tuberculosis. Antimicrobial Agents and Chemotherapy 48, 3175-3178 (2004).

40. Zaunbrecher, M. A., Sikes, R. D., Metchock, B., Shinnick, T. M. \& Posey, J. E. Overexpression of the chromosomally encoded aminoglycoside acetyltransferase eis confers kanamycin resistance in Mycobacterium tuberculosis. Proceedings of the National Academy of Sciences 106, 20004-20009 (2009).

41. Awasthy, D., Bharath, S., Subbulakshmi, V. \& Sharma, U. Alanine racemase mutants of Mycobacterium tuberculosis require D-alanine for growth and are defective for survival in macrophages and mice. Microbiology 158, 319-327 (2012).

42. Köser, C. U. et al. Whole-genome sequencing for rapid susceptibility testing of M. tuberculosis. The New England journal of medicine 369, 290-2 (2013).

43. Schön, T. et al. Mycobacterium tuberculosis drug-resistance testing: challenges, recent developments and perspectives. Clinical Microbiology and Infection 23, 154160 (2017).

44. Smith, T., Wolff, K. A. \& Nguyen, L. in Current topics in microbiology and immunology 374, 53-80 (NIH Public Access, 2012).

45. McNerney, R. et al. Removing the bottleneck in whole genome sequencing of Mycobacterium tuberculosis for rapid drug resistance analysis: a call to action. International Journal of Infectious Diseases 56, 130-135 (2017). 


\section{FIGURE LEGENDS}

Figure 1. Geographical distribution of the 6,465 Mycobacterium tuberculosis isolates analysed in the study

This world map shows the main geographical origins of the M. tuberculosis isolates included in this study. The study comprises strains from more than 30 countries, of which the 18 major contributors are shown on this map. See Supplementary table 1 for a detailed description of each dataset. Inner pie charts show the proportion of each of the main four lineages, and the outer charts summarise the drug resistance phenotypes. 'Drug-resistant' refers to non-MDRTB/XDR-TB resistance.

Figure 2. Whole genome phylogeny of the 6,465 M. tuberculosis isolates

Maximum likelihood phylogenetic tree constructed using 102,160 SNPs and 11,122 insertions and deletions spanning the whole genome and rooted on $M$. canetti (not shown), colourcoded by lineage (inner circle) and drug resistance status (outer circle). 'Susceptible' refers to isolates being susceptible to all drugs tested. 'Drug-resistant' refers to strains being resistant to multiple drugs but not classified as multidrug-resistant (MDR-TB) or extensively drugresistant XDR-TB.

\section{Figure 3}

(Log) Odds ratios from SNP-drug resistance associations are a potential surrogate for resistance level. (A) Within each drug, boxplots for the log odds ratios $\left(P<1 \times 10^{-5}\right)$ for each gene are arranged by increasing median values (as indicated by the horizontal line in the boxes) to show their relative effect on resistance. Mutations known to confer low, 
intermediate or high levels of resistance (See Online Methods) are represented by points coloured blue, yellow or red, respectively, and their size is proportional to their frequency; overall, higher levels of resistance are reflected by higher odds ratios; one exception is for $r$ rs and CAP, where the $\mathrm{G} 1484 \mathrm{C} / \mathrm{T}$ (high level resistance) mutation has a lower odds ratio than A1401G (intermediate level) due to its low frequency; a similar effect is seen for the same G1484C/T mutation in KAN resistance; $(B)$ The distribution of $(\log )$ odds ratios $\left(P<1 \times 10^{-5}\right)$ for the mutations within unknown, or known low (blue), intermediate (yellow) or high (red) levels of resistance; (C) The distribution of ( $\log$ ) odds ratios for known and novel drug resistance mutations $\left(\mathrm{P}<1 \times 10^{-5}\right)$. All boxplots consist of boxes (median and interquartile range) and whiskers that extend to the most extreme data point which is no more than 1.5 times the interquartile range from the box. 
Table 1

MDR-TB and XDR-TB gene-based associations

\begin{tabular}{|c|c|c|c|c|c|c|c|}
\hline Comparison & Rv number & Gene name & P-value & $\begin{array}{l}\text { NS } \\
\text { SNPs }\end{array}$ & $\begin{array}{l}\text { Indels } \\
\text { (frame.) }\end{array}$ & $\begin{array}{l}\text { Assoc. } \\
\text { SNPs }\end{array}$ & $\begin{array}{l}\text { PhyC } \\
\text { SNPs }\end{array}$ \\
\hline MDR-TB vs. Susc. & $R v 0667$ & $r p o B$ & $2.99 \mathrm{E}-103$ & 159 & $7(0)$ & 6 & 33 \\
\hline MDR-TB vs. Susc. & $R v 1908 c$ & katG & $2.44 \mathrm{E}-65$ & 177 & $12(9)$ & 2 & 8 \\
\hline MDR-TB vs. Susc. & $R v 1482 c-R v 1483$ & $R v 1482 c-f a b G 1$ & $1.28 \mathrm{E}-17$ & 8 & 0 & 1 & 4 \\
\hline MDR-TB vs. Susc. & $R v 2427 A-R v 2428$ & $o x y R^{\prime}-a h p C$ & $5.26 \mathrm{E}-15$ & 17 & 3 & 0 & 7 \\
\hline MDR-TB vs. Susc. & Rv3919c & gid & $1.09 \mathrm{E}-08$ & 137 & $26(26)$ & 0 & 15 \\
\hline MDR-TB vs. Susc. & $R v 1484$ & $\operatorname{inh} A$ & $8.55 E-07$ & 9 & 0 & 0 & 3 \\
\hline MDR-TB vs. Susc. & $R v 0682$ & $r p s L$ & 7.31E-06 & 6 & 0 & 0 & 2 \\
\hline XDR-vs. MDR-TB & $R v 0006$ & gyrA & $2.46 \mathrm{E}-37$ & 147 & 0 & 4 & 5 \\
\hline XDR- vs. MDR-TB & $r r s$ & $r r s$ & $4.33 \mathrm{E}-17$ & 91 & 4 & 1 & 5 \\
\hline XDR-vs. MDR-TB & $R v 3806 c$ & ubiA & $4.22 \mathrm{E}-07$ & 47 & 0 & 1 & 1 \\
\hline XDR- vs. MDR-TB & Rv3793-Rv3794 & embC-embA & $8.73 \mathrm{E}-06$ & 6 & 6 & 0 & 6 \\
\hline XDR-TB vs. Susc. & $R v 0667$ & $r p o B$ & 4.13E-183 & 159 & $7(0)$ & 5 & 3 \\
\hline XDR-TB vs. Susc. & $R v 3795$ & $e m b B$ & $1.54 \mathrm{E}-75$ & 168 & $2(0)$ & 4 & 2 \\
\hline XDR-TB vs. Susc. & $R v 2043 c$ & $p n c A$ & $4.33 E-65$ & 117 & $25(22)$ & 1 & 9 \\
\hline XDR-TB vs. Susc. & $R v 1908 c$ & katG & $9.52 E-60$ & 177 & $12(9)$ & 1 & 1 \\
\hline XDR-TB vs. Susc. & Rv3793-Rv3794 & embC-embA & $1.07 \mathrm{E}-31$ & 6 & 6 & 2 & 4 \\
\hline XDR-TB vs. Susc. & $r r s$ & $r r s$ & $5.14 \mathrm{E}-28$ & 91 & 4 & 2 & 3 \\
\hline XDR-TB vs. Susc. & $R v 1482 c-R v 1483$ & $R v 1482 c-f a b G 1$ & $1.98 \mathrm{E}-27$ & 8 & 0 & 2 & 1 \\
\hline XDR-TB vs. Susc. & $R v 1484$ & inhA & $3.09 E-26$ & 9 & 0 & 1 & 1 \\
\hline XDR-TB vs. Susc. & $R v 0006$ & gyrA & $8.62 E-26$ & 147 & 0 & 4 & 5 \\
\hline XDR-TB vs. Susc. & $R v 0668$ & rpoC & $2.62 \mathrm{E}-21$ & 153 & $1(0)$ & 1 & 9 \\
\hline XDR-TB vs. Susc. & $R v 0682$ & $r p s L$ & $2.02 \mathrm{E}-18$ & 6 & 0 & 1 & 3 \\
\hline XDR-TB vs. Susc. & $R v 3144 c-R v 3145$ & PPE52-nuoA & $3.65 \mathrm{E}-11$ & 24 & 1 & 1 & 2 \\
\hline XDR-TB vs. Susc. & $R v 3854 c$ & ethA & $1.80 \mathrm{E}-10$ & 163 & $38(35)$ & 0 & 1 \\
\hline XDR-TB vs. Susc. & $R v 2936$ & $d r r A$ & $1.46 \mathrm{E}-08$ & 19 & 0 & 1 & 9 \\
\hline XDR-TB vs. Susc. & $R v 2416 c-R v 2417 c$ & $e i s-R v 2417 c$ & $2.53 \mathrm{E}-07$ & 12 & 1 & 0 & 3 \\
\hline XDR-TB vs. Susc. & $R v 1144-R v 1145$ & Rv1144-mmpL13a & $1.48 \mathrm{E}-07$ & 33 & 4 & 1 & 2 \\
\hline XDR-TB vs. Susc. & $R v 3854 c-R v 3855$ & ethA-ethR & $9.87 \mathrm{E}-06$ & 12 & 0 & 1 & 0 \\
\hline
\end{tabular}

This table shows loci (protein and RNA coding regions, intergenic regions) associated with MDR- and XDR-TB resistance $\left(\mathrm{P}<1 \times 10^{-5}\right)$. The column labelled as 'NS SNPs' shows the number of non-synonymous SNPs in the genes; the column 'Indels (frame.)' refers to the number of small indels resulting in frameshifts in the genes; 'Assoc. SNPs' refers to the number of SNPs identified by GWAS and 'PhyC SNPs' is the number of homoplastic SNPs identified using the PhyC test. The PhyC test additionally detected folC, pncA-Rv2044c and whiB6-Rv3863 loci when comparing MDR-TB against the susceptible group; and eis-Rv2417c, gyrB, rrs, folC, alr, gid, and the thyX-hsdS.1 intergenic region when comparing XDR-TB against MDR-TB; and alr, gyrB, pyrG, rpoA, and thyX-hsdS.1 loci when comparing XDR-TB against susceptible. Similarly, GWAS using SNPs additionally identified embC-embA for MDR-TB vs susceptible (1 SNP), rrs and ubiA genes for XDR-TB vs MDR-TB (each 1 SNP), and the ubiA gene for XDR-TB vs. susceptible (2 SNPs). 
Table 2

Individual drug gene-based associations in the complete dataset

\begin{tabular}{|c|c|c|c|c|c|c|c|}
\hline Drug* & Rv number & Gene name & P-value & $\begin{array}{c}\text { NS } \\
\text { SNPs }\end{array}$ & $\begin{array}{c}\text { Indels } \\
\text { (frame.) }\end{array}$ & $\begin{array}{l}\text { Assoc. } \\
\text { SNPs }\end{array}$ & $\begin{array}{l}\text { Phyc } \\
\text { SNPs }\end{array}$ \\
\hline Isoniazid & $R v 1908 c$ & katG & $1.02 \mathrm{E}-112$ & 177 & $12(9)$ & 1 & 3 \\
\hline Isoniazid & $R v 1482 c-R v 1483$ & $R v 1482 c-f a b G 1$ & $5.41 E-54$ & 8 & 0 & 2 & 2 \\
\hline Isoniazid & $R v 2427 A-R v 2428$ & oxy $R^{\prime}-a h p C$ & $8.51 \mathrm{E}-27$ & 17 & 3 & 0 & 3 \\
\hline Isoniazid & $R v 1484$ & $\operatorname{inh} A$ & $3.29 \mathrm{E}-07$ & 9 & 0 & 1 & 1 \\
\hline Rifampicin & $R v 0667$ & rрoB & $8.47 E-226$ & 159 & $7(0)$ & 7 & 9 \\
\hline Rifampicin & $R v 0668$ & rpoC & $2.57 \mathrm{E}-08$ & 153 & $1(0)$ & 0 & 9 \\
\hline Ethambutol & Rv3795 & $e m b B$ & $2.48 \mathrm{E}-129$ & 168 & $2(0)$ & 4 & 10 \\
\hline Ethambutol & Rv3793-Rv3794 & $e m b C-e m b A$ & $8.49 E-42$ & 6 & 6 & 2 & 5 \\
\hline Ethambutol & $R v 3806 c$ & ubiA & $3.93 \mathrm{E}-13$ & 47 & 0 & 1 & 2 \\
\hline Ethambutol & $R v 2820 c$ & . & $2.55 \mathrm{E}-08$ & 16 & 0 & 1 & 0 \\
\hline Ethambutol & $R v 3300 c$ & . & $1.33 \mathrm{E}-07$ & 39 & $5(3)$ & 0 & 0 \\
\hline Ethionamide & $R v 1482 c-R v 1483$ & $R v 1482 c-f a b G 1$ & $6.01 \mathrm{E}-16$ & 8 & 0 & 2 & 2 \\
\hline Ethionamide & $R v 1484$ & $\operatorname{inh} A$ & $6.72 \mathrm{E}-07$ & 9 & 0 & 1 & 0 \\
\hline Pyrazinamide & $R v 2043 c$ & $p n c A$ & $3.62 E-99$ & 117 & $25(22)$ & 2 & 1 \\
\hline Pyrazinamide & $R v 2043 c-R v 2044 c$ & $p n c A-R v 2044 c$ & $6.64 \mathrm{E}-30$ & 4 & 1 & 1 & 1 \\
\hline Streptomycin & $R v 0682$ & $r p s L$ & $2.67 \mathrm{E}-85$ & 6 & 0 & 2 & 2 \\
\hline Streptomycin & $R v 3919 c$ & gid & $3.54 \mathrm{E}-26$ & 137 & $26(26)$ & 0 & 1 \\
\hline Streptomycin & $r r s$ & rrs & $3.95 \mathrm{E}-13$ & 91 & 4 & 1 & 3 \\
\hline Amikacin & rrs & rrs & $5.28 \mathrm{E}-48$ & 91 & 4 & 1 & 1 \\
\hline Kanamycin & $r r s$ & $r r s$ & $1.76 \mathrm{E}-48$ & 91 & 4 & 2 & 2 \\
\hline Kanamycin & $R v 2416 c-R v 2417 c$ & $e i s-R v 2417 c$ & $9.84 \mathrm{E}-21$ & 12 & 1 & 1 & 1 \\
\hline Capreomycin & rrs & $r r s$ & $1.68 \mathrm{E}-39$ & 91 & 4 & 1 & 1 \\
\hline Capreomycin & $R v 2172 c-R v 2173$ & Rv2172c-idsA2 & $7.18 \mathrm{E}-06$ & 18 & 0 & 0 & 0 \\
\hline Ciprofloxacin & $R v 0006$ & gyrA & $4.48 E-45$ & 147 & 0 & 2 & 2 \\
\hline Moxifloxacin & $R v 0006$ & gyrA & $2.98 \mathrm{E}-23$ & 147 & 0 & 3 & 5 \\
\hline Ofloxacin & $R v 0006$ & gyrA & $4.87 \mathrm{E}-115$ & 147 & 0 & 4 & 6 \\
\hline D-Cycloserine & $R v 3423 c$ & $a l r$ & $1.23 \mathrm{E}-13$ & 57 & 0 & 1 & 0 \\
\hline D-Cycloserine & $R v 0342$ & iniA & $3.36 \mathrm{E}-08$ & 76 & $13(12)$ & 1 & 0 \\
\hline PAS & $R v 2764 c$ & thyA & $3.74 \mathrm{E}-10$ & 36 & $4(4)$ & 0 & 0 \\
\hline PAS & $R v 2754 c-R v 2755 c$ & thyX-hsdS.1 & 4.27E-07 & 21 & 0 & 1 & 1 \\
\hline
\end{tabular}

This table shows loci (protein and RNA coding and intergenic regions) associated with resistance to individual drugs $\left(\mathrm{P}<1 \times 10^{-5}\right)$. The column labelled as 'NS SNPs' shows the number of nonsynonymous SNPs in the genes; the column 'Indels (frame.)' refers to the number of small indels resulting in frameshifts in the genes; 'Assoc. SNPs' is the number of SNPs identified by GWAS, and 'PhyC SNPs' refers to the number of homoplastic SNPs identified using the PhyC test. * The GWAS additionally detected a significant association of a SNP (C213R) in the Rv2688c locus (known efflux gene) with Moxifloxacin and Fluoroquinolones; the PhyC test additionally detected other associated loci for Amikacin (eis-Rv2417c), Capreomycin and D-Cycloserine (Ihr), Kanamycin (thyXhsdS.1), Rifampicin (rpoA). Abbreviations: PAS, para-aminosalicylic acid. 
Table 3

Impact on drug resistance prediction (\%) from GWAS findings

\begin{tabular}{lcccccccc}
\hline \multirow{2}{*}{ Drug } & \multicolumn{2}{c}{ TBDR panel } & \multicolumn{3}{c}{ + SNPs } & \multicolumn{2}{c}{ + small indels + } & \multicolumn{2}{c}{ + big deletions + } \\
& Sens. & Spec. & Sens. & Spec. & Sens. & Spec. & Sens. indels + SNPs & Spec. \\
\hline Isoniazid & 89 & 97 & 89 & 97 & $\mathbf{9 0}$ & 97 & 90 & 97 \\
Rifampicin & 92 & 98 & 92 & 98 & $\mathbf{9 3}$ & 98 & 93 & 98 \\
Ethambutol & 90 & 92 & 90 & 92 & 90 & 92 & 90 & 92 \\
Ethionamide & 64 & $\mathbf{7 8}$ & $\mathbf{7 8}$ & $\mathbf{7 4}$ & $\mathbf{8 4}$ & $\mathbf{7 2}$ & $\mathbf{8 8}$ & 72 \\
Pyrazinamide & 52 & 98 & 52 & 98 & $\mathbf{6 3}$ & 97 & $\mathbf{6 5}$ & 97 \\
Streptomycin & $\mathbf{7 6}$ & 93 & 76 & 93 & $\mathbf{8 0}$ & 91 & 80 & 91 \\
Amikacin & 83 & 96 & 83 & 96 & $\mathbf{8 5}$ & 93 & 85 & 93 \\
Kanamycin & 84 & 98 & 84 & 98 & 84 & 98 & 84 & 98 \\
Capreomycin & $\mathbf{7 5}$ & 96 & 75 & 96 & $\mathbf{8 1}$ & 95 & 81 & 95 \\
Ciprofloxacin & 89 & 98 & 89 & 98 & 89 & 98 & 89 & 98 \\
Moxifloxacin & 85 & 90 & 85 & 90 & 85 & 90 & 85 & 90 \\
Ofloxacin & 86 & 96 & 86 & 96 & 86 & 96 & 86 & 96 \\
D-Cycloserine & - & - & $\mathbf{5 5}$ & $\mathbf{9 2}$ & $\mathbf{6 1}$ & 90 & 61 & 90 \\
PAS & 10 & 100 & $\mathbf{2 0}$ & $\mathbf{9 9}$ & $\mathbf{4 0}$ & 94 & $\mathbf{6 5}$ & 94 \\
\hline MDR-TB & 87 & 100 & 87 & 100 & $\mathbf{8 8}$ & 100 & $\mathbf{8 9}$ & 100 \\
XDR-TB & $\mathbf{7 7}$ & 99 & $\mathbf{7 8}$ & $\mathbf{9 9}$ & $\mathbf{7 9}$ & 98 & 79 & 98 \\
\hline
\end{tabular}

This table shows the sensitivity and specificity achieved by known drug resistance SNPs and indels (TBDR, tbdr.lshtm.ac.uk) 9, 31 when predicting phenotypic drug resistance ("TBDR panel" columns). The SNPs in the TBDR contribute $100 \%$ to the stated sensitivity, except rifampicin $(99.8 \%)$ and ethionamide (99.3\%). The other columns show the improvements achieved when including the SNPs, small indels and large deletions found associated with drug resistance in this study. The improvements in sensitivity are highlighted in grey. Abbreviations: MDR-TB, multidrug-resistant; PAS Para-aminosalicylic acid; Sens., sensitivity; Spec., specificity; SNPs, single nucleotide polymorphisms; XDR-TB, extensively drug-resistant. 


\section{ONLINE METHODS}

\section{Sequence data and variant calling}

Sequence data for 6,465 Mycobacterium tuberculosis complex clinical isolates were generated as part of a collaborative global drug resistance project $(n=2,637$, pathogenseq.Ishtm.ac.uk) or downloaded from the public domain $(n=3,828)$ (Supplementary table 1). All isolates had undergone drug susceptibility testing by phenotypic methods. These isolates represented multiple populations from different geographic areas, and all four main lineages (1 to 4) (Supplementary table 1 ). The 2,637 samples not previously sequenced were Illumina sequenced generating paired-end reads of at least $50 \mathrm{bp}$ with at least 50 -fold genome coverage. The analytical workflow for the raw sequence data is summarised in Supplementary figure 5. The new and archived raw sequence data were aligned to the H37Rv reference genome (Genbank accession number: NC_000962.3) using the BWA mem algorithm ${ }^{46}$ (settings: -c $100-$ T 50). The SAMtools/BCFtools ${ }^{47}$ (default settings) and GATK ${ }^{48}$ software were used to call SNPs and small indels. The GATK parameters used are "-T UnifiedGenotyper -ploidy 1 -glm BOTH -allowPotentiallyMisencodedQuals 2". The overlapping set of variants from the two algorithms was retained for further analysis. Alleles were additionally called across the whole genome (including SNP sites) using a coveragebased approach ${ }^{5,49}$. A missing call was assigned if the total depth of coverage at a site did not reach a minimum of 20 reads or none of the four nucleotides accounted for at least $75 \%$ of the total coverage. Samples or SNP sites having an excess of $10 \%$ missing genotype calls were removed. This quality control step was implemented to remove samples with bad quality genotype calls due to poor depth of coverage or mixed infections. The final dataset included 6,465 isolates and 102,160 genome-wide SNPs. Delly2 software ${ }^{50}$ was used to identify large 
deletions. All large deletions were confirmed using localised de novo assembly, and those found in association analysis ( $d f r A /$ thy $A, p n c A$, ethA/ethR, katG) confirmed using PCR.

\section{Phenotypic drug susceptibility testing}

Drug susceptibility data was obtained from World Health Organisation recognised testing protocols ${ }^{51}$. The M. tuberculosis (Mtb) isolates that provided sequence data included in this study are summarised in Supplementary table 1. Each sequence included in the study was derived from an isolate from an individual patient. Some DNA samples were from archived stocks (e.g. India, collected prior to 2009 and Malawi, collected between 1996 and 2010) and others were extracted specifically for this study. Information regarding isolates with previously reported sequence data was derived from published materials. Isolates were classed as resistant or susceptible to a drug on the basis of phenotypic testing using either the BACTEC 460 TB System (Becton Dickinson), the BACTEC Mycobacterial Growth Indicator Tube (MGIT) 960 system (Becton Dickinson) ${ }^{52}$, solid agar or Lowenstein Jensen slopes ${ }^{53,54}$. Not all samples were tested for resistance to all drugs, most notably some isolates found susceptible to the first-line drugs were not subjected to testing for resistance to second-line drugs. Where isolates were not tested for resistance to a particular drug they were excluded from the analysis for that drug. Drug susceptibility testing was mainly undertaken in local laboratories participating in the WHO supranational laboratory network using the recognised testing protocols ${ }^{51}$. Isolates from Malawi were shipped to the United Kingdom's Mycobacterium Reference Laboratory for testing. Isolates from Uganda were tested at the Joint Clinical Research Centre (JCRC) in Kampala with quality control performed by the US Centers for Disease Control and Prevention (CDC). The Peruvian isolates were initially tested for resistance to rifampicin and isoniazid using the Microscopic Observation Drug 
Susceptibility assay (MODS) ${ }^{54}$ at the Universidad Peruana Cayetano Heredia (UPCH) prior to transfer to the national reference laboratory for further testing. In Peru susceptibility to pyrazinamide (PZA) was assessed by the Wayne assay; a colorimetric biochemical test during which PZA is hydrolysed to free pyrazinoic acid ${ }^{55}$. Testing using the BACTEC $960^{\circledR} \mathrm{MGIT}^{\circledR}$ or BACTEC $460^{\circledR}$ (Becton-Dickinson ${ }^{\circledR}$ ) was performed according to the manufacturer's indications ${ }^{56}$. Pyrazinamide sensitivity was determined by using BACTEC 7H12 liquid medium, $\mathrm{pH}$ 6.0, at $100 \mu \mathrm{g} / \mathrm{mL}$ (BACTEC PZA test medium, Becton Dickinson). When testing on agar critical drug concentrations used were rifampicin $1 \mu \mathrm{g} / \mathrm{mL}$, isoniazid $0.2 \mu \mathrm{g} / \mathrm{mL}$, streptomycin $2 \mu \mathrm{g} / \mathrm{mL}$, and ethambutol $5 \mu \mathrm{g} / \mathrm{mL}$, ciprofloxacin $2 \mu \mathrm{g} / \mathrm{mL}$, amikacin $5 \mu \mathrm{g} / \mathrm{mL}$, capreomycin 10 $\mu \mathrm{g} / \mathrm{mL}$, kanamycin $5 \mu \mathrm{g} / \mathrm{mL}$ (Pakistan $6 \mu \mathrm{g} / \mathrm{mL}$ ), ethionamide $5 \mu \mathrm{g} / \mathrm{mL}$ and para-aminosalicylic acid $2 \mu \mathrm{g} / \mathrm{mL}^{53}$. For Lowenstein-Jensen drug concentrations used were for streptomycin 4.0 $\mu \mathrm{g} / \mathrm{ml}$, isoniazid $0.2 \mu \mathrm{g} / \mathrm{ml}$, rifampicin $40.0 \mu \mathrm{g} / \mathrm{ml}$, ethambutol $2.0 \mu \mathrm{g} / \mathrm{ml}$, capreomycin 40.0 $\mu \mathrm{g} / \mathrm{ml}$, kanamycin $30.0 \mu \mathrm{g} / \mathrm{ml}$ (China) or $20.0 \mu \mathrm{g} / \mathrm{ml}$ (Vietnam), ofloxacin $2.0 \mu \mathrm{g} / \mathrm{ml}$, ethionamide $40 \mu \mathrm{g} / \mathrm{ml}$, thioacetone $(10 \mu \mathrm{g} / \mathrm{ml})$, pyrazinamide $200 \mu \mathrm{g} / \mathrm{ml}$, cycloserine 30 $\mu \mathrm{g} / \mathrm{ml}$ and para-aminosalicylic acid (PAS) $0.5 \mu \mathrm{g} / \mathrm{ml}^{55}$.

\section{Phylogenetic tree and association analysis}

The best-scoring maximum likelihood phylogenetic tree rooted on Mycobacterium canettii (Genbank accession number: HE572590) was constructed by $R A x M L$ software $^{57}(10,000$ bootstrap samples) using the 102,160 high quality SNP sites. Spoligotypes were inferred in silico using SpolPred ${ }^{58}$, and strain-types determined using lineage-specific SNPs ${ }^{5}$. Further population structure assessment was performed using principal components analysis (Supplementary figure 2), which clustered samples by genotype congruent with the phylogenetic tree. The principal components were calculated from a SNP pair-wise distance matrix between each sample, and the first five components (summarising $82.7 \%$ of genetic 
variation) were used as covariates in the regression-based association models. Mixed regression models were employed to estimate the strength of association between the binary drug resistance outcome (resistance vs. susceptible) and the aggregate number of mutations (SNPs, indels or large deletions) by coding region, RNA loci and intergenic regions, as well as operons $^{49}$. The low frequency of variants required the aggregation of mutations to increase the power of detecting associated loci, and a mixed model approach has been demonstrated to work well at adjusting for the confounding effects of Mtb lineage, sub-lineage and outbreak-based population structure. ${ }^{49}$ The operons or functional units containing clusters of genes under the control of the same promoter were determined from TBDB (see URLs). Gene function was extracted from the Tuberculist webserver (see URLS). The mixed models also included the principal components to account for the main Mtb lineage and sub-lineage effects, and a SNP inferred kinship matrix as a random effect to account for highly related samples and fine-scale population structure due to potential outbreaks ${ }^{49}$. These models were implemented in GEMMA (v.1.1.2) software ${ }^{59}$. A SNP-based GWAS was used to identify individual variants associated with drug resistance expected to fall within the genes found associated in the 'main' analysis. To minimise any co-resistance between drugs, we adjusted for the presence of other resistance in the regression models. Co-resistance is expected to result from exposure to multiple anti-tuberculous drugs and the step-wise accumulation of mutations. Statistical significance thresholds to account for multiple testing were established using a permutation approach that sorted phenotypic test data without replacement and reperformed GWAS analysis (10,000 times). We report all findings that are below a calculated permutation threshold of $\mathrm{P}<1 \times 10^{-5}$. All statistical analyses were performed using $\mathrm{R}$ software. To identify SNPs enriched by convergent evolution and provide further evolutionary evidence, the phylogenetic-based phyC approach was employed ${ }^{9}$ using the implementation made 
available in a previous study ${ }^{60}$. Any potential co-resistance effects were dissected through consulting gene annotation and published literature to report the most plausible role in drug resistance. Additionally, long branches in the phylogenetic tree leading up to clades enriched with drug resistant isolates leads to spurious associations. Truly drug resistant mutations often originate multiple times independently in the phylogeny. Mutations which originated once in the tree (i.e. clade-specific mutations), which are likely to lead to spurious associations, were removed from the GWAS results.

\section{Detection of putative compensatory mechanisms}

Loci were identified as being putative compensatory if they: (i) were associated with drug resistance, (ii) harboured homoplastic mutations, (iii) shared a similar biological function with a known drug-target or drug-activating enzyme, and (iv) were significantly more mutated in the presence of mutations in the drug-target or drug-activating enzyme coding gene. In the latter, deep phylogenetic and synonymous SNPs were removed prior to calculating the number of samples with non-synonymous SNPs at genes of interest (e.g. Ala1075Ala at rpoB or Glu1092Asp at $r p o C)$. The significance of differences between studied genes was calculated using Fisher's exact test (cut-off of $\mathrm{P}<1 \times 10^{-8}$ ).

\section{Protein mutation modelling}

Apo crystal structures for alr were downloaded from the Protein Data Bank (PDBe1XFC ${ }^{61}$ ) and then subjected to modelling of missing residues, WinCOOT regularisation, and removal of pyridoxal 5'-phosphate from both chains. The mCSM and DUET web servers were used to assess changes in protein stability, mCSM-PPI to quantify effects on protein-protein interactions and mCSM-Lig to quantify effects on drug binding ${ }^{62-64}$. For ligand binding, DCycloserine was docked in the active site using Autodock Vina and Gold software ${ }^{65,66}$.

\section{Statistical analyses}


The statistical mixed models used for association analysis are described above. The terms 'low', 'intermediate' or 'high' levels of resistance referred to in the text and Figure $\mathbf{3}$ denote whether a mutation is known to confer low, intermediate or high MIC values, respectively, as reported in the literature ${ }^{18,40,67-71}$. Wilcoxon tests and linear regression models were used to compare differences in (log) odds ratios between resistance levels. Samples which had more than one known resistance causing variant were removed from these calculations. $\mathrm{R}$ statistical software (v3.4.1; see URLs) was used to perform this analysis. The R library "maps" was used to generate the world map with lineage and drug resistance frequencies.

\section{DATA AVAILABILITY}

All raw sequencing data are available, and the study accession numbers are listed in

Supplementary table 1. For samples sequenced as part of our collaborative global drug resistance project, the ENA accession numbers for the isolates and their phenotypic drug susceptibility data are provided in Supplementary data 2.

\section{URLS}

The TubercuList knowledge base, http://tuberculist.epfl.ch; Tuberculosis Database, http://www.tbdb.org; R Statistical software, https://www.r-project.org; TB Global Drug Resistance Collaboration, http://pathogenseq.Ishtm.ac.uk/\#tuberculosis):

\section{METHODS-ONLY REFERENCES}

46. $\mathrm{Li}, \mathrm{H}$. Toward better understanding of artifacts in variant calling from high-coverage samples. Bioinformatics 30, 2843-2851 (2014).

47. Li, H. et al. The Sequence Alignment/Map format and SAMtools. Bioinformatics 25, 2078-2079 (2009).

48. DePristo, M. A. et al. A framework for variation discovery and genotyping using nextgeneration DNA sequencing data. Nature genetics 43, 491-8 (2011). 
49. Phelan, J. et al. Mycobacterium tuberculosis whole genome sequencing and protein structure modelling provides insights into anti-tuberculosis drug resistance. $B M C$ medicine 14, 31 (2016).

50. Rausch, T. et al. DELLY: Structural variant discovery by integrated paired-end and split-read analysis. Bioinformatics 28, i333-i339 (2012).

51. World Health Organization. WHO | Guidelines for surveillance of drug resistance in tuberculosis. (2009).

52. Kubica, G. \& Kent, K. Public health mycobacteriology: a guide for the level III laboratory. Centers for Disease Control, U.S. Department of Health and Human Services, Atlanta, GA 60-63 (1985).

53. Canetti, G. et al. Mycobacteria: Laboratory Methods For Testing Drug Sensitivity and Resistance. Bulletin of the World Health Organization 29, 565-78 (1963).

54. Minion, J., Leung, E., Menzies, D. \& Pai, M. Microscopic-observation drug susceptibility and thin layer agar assays for the detection of drug resistant tuberculosis: a systematic review and meta-analysis. The Lancet Infectious Diseases 10, 688-698 (2010).

55. Wayne, L. G. Simple pyrazinamidase and urease tests for routine identification of mycobacteria. The American review of respiratory disease 109, 147-51 (1974).

56. Pfyffer, G. E., Palicova, F., Sabine Rüsch-Gerdes S. Testing of Susceptibility of Mycobacterium tuberculosis to Pyrazinamide with the Nonradiometric BACTEC MGIT 960 System J Clin Microbiol. 40, 1670-4 (2002).

57. Stamatakis, A., Hoover, P. \& Rougemont, J. A rapid bootstrap algorithm for the RAxML Web servers. Syst. Biol. 57, 758-71 (2008).

58. Coll, F. et al. SpolPred: rapid and accurate prediction of Mycobacterium tuberculosis spoligotypes from short genomic sequences. Bioinformatics 28, 2991-3 (2012).

59. Zhou, X. \& Stephens, M. Genome-wide efficient mixed-model analysis for association studies. Nature genetics 44, 821-4 (2012).

60. Alam, M. T. et al. Dissecting vancomycin-intermediate resistance in staphylococcus aureus using genome-wide association. Genome biology and evolution 6, 1174-85 (2014).

61. Velankar, S. et al. PDBe: improved accessibility of macromolecular structure data from PDB and EMDB. Nucleic Acids Research 44, D385-D395 (2016).

62. Pires, D. E. V, Ascher, D. B. \& Blundell, T. L. mCSM: predicting the effects of mutations in proteins using graph-based signatures. Bioinformatics 30, 335-342 (2014).

63. Pires, D. E. V., Blundell, T. L. \& Ascher, D. B. mCSM-lig: quantifying the effects of mutations on protein-small molecule affinity in genetic disease and emergence of drug resistance. Scientific Reports 6, 29575 (2016).

64. Pires, D. E. V, Ascher, D. B. \& Blundell, T. L. DUET: a server for predicting effects of mutations on protein stability using an integrated computational approach. Nucleic Acids Research 42, W314-W319 (2014).

65. Trott, O. \& Olson, A. J. Software news and update AutoDock Vina: Improving the speed and accuracy of docking with a new scoring function, efficient optimization, and multithreading. Journal of Computational Chemistry 31, 455-461 (2010).

66. Verdonk, M. L. et al. Modeling water molecules in protein-ligand docking using GOLD. Journal of Medicinal Chemistry 48, 6504-6515 (2005).

67. Wong, S. Y. et al. Mutations in gidB Confer Low-Level Streptomycin Resistance in Mycobacterium tuberculosis. Antimicrobial Agents and Chemotherapy 55, 2515-2522 
(2011).

68. Rueda, J. et al. Genotypic Analysis of Genes Associated with Independent Resistance and Cross-Resistance to Isoniazid and Ethionamide in Mycobacterium tuberculosis Clinical Isolates. Antimicrobial Agents and Chemotherapy 59, 7805-7810 (2015).

69. Kambli, P. et al. Correlating rrs and eis promoter mutations in clinical isolates of Mycobacterium tuberculosis with phenotypic susceptibility levels to the second-line injectables. International Journal of Mycobacteriology 5, 1-6 (2016).

70. Domínguez, J. et al. Clinical implications of molecular drug resistance testing for Mycobacterium tuberculosis; a TBNET/RESIST-TB consensus statement. Int. J. Tuberc. Lung Dis. 20, 24-42 (2016).

71. Cambau, E. et al. Revisiting susceptibility testing in MDR-TB by a standardized quantitative phenotypic assessment in a European multicentre study. J. Antimicrob. Chemother. 70, 686-696 (2015). 